OPEN ACCESS

Edited by:

Tangxin Xiao,

Changzhou University, China

Reviewed by:

Subhamay Pramanik,

University of Kansas, United States

Christopher Pigge,

The University of lowa, United States

Luiz Fernando Cappa De Oliveira, Juiz de Fora Federal University, Brazil

*Correspondence:

Eli Zysman-Colman

eli.zysman-colman@st-

andrews.ac.uk

tThese authors have contributed equally to this work

Specialty section: This article was submitted to

Supramolecular Chemistry,

a section of the journa

Frontiers in Chemistry

Received: 22 June 2021

Accepted: 12 July 2021

Published: 02 August 2021

Citation:

Mackenzie CFR, Delforce L, Rota Martir D, Cordes DB, Slawin AMZ and Zysman-Colman E (2021) A Luminescent 1D Silver Polymer

Containing [2.2]

Paracyclophane Ligands.

Front. Chem. 9:728845

doi: 10.3389/fchem.2021.728845

\section{A Luminescent 1D Silver Polymer Containing [2.2]Paracyclophane Ligands}

\author{
Campbell F. R. Mackenzie ${ }^{\dagger}$, Lucie Delforce ${ }^{\dagger}$, D. Rota Martir, David B. Cordes, \\ Alexandra M. Z. Slawin and Eli Zysman-Colman* \\ Organic Semiconductor Centre, EaStCHEM School of Chemistry, University of St Andrews, Fife, United Kingdom
}

[2.2]Paracyclophane scaffolds have seen limited use as building blocks in supramolecular chemistry. Here, we report the synthesis and characterization of a 1D coordination polymer consisting of silver(I) ions bound to a [2.2]paracyclophane scaffold functionalized with two 4-pyridyl units. The structure of the polymer has been determined from single crystal X-ray diffraction analysis and reveals two different silver coordination motifs that alternate along the 1D coordination polymer. The coordination polymer exhibits strong blue and sky-blue fluorescence in solution and in the crystalline solid state, respectively.

Keywords: coordination polymer, cyclophane, supramolecular chemistry, silver(I), paracyclophane

\section{INTRODUCTION}

Coordination polymers are polymeric arrays of monomeric units that are held together through metal coordination (Yang et al., 2015). These materials continue to attract attention due to their use in a wide range of applications from medicine (Medici et al., 2016), to catalysis (Zeng et al., 2016), sensors (Yi et al., 2016) and luminescent materials (Yersin et al., 2017) (To et al., 2020). Among the metals used to synthesize coordination polymers, silver is of particular interest due to the range of geometries and coordination modes available (Young and Hanton, 2008). Silver has been demonstrated to form coordination polymers with a range of morphologies, $1 \mathrm{D}, 2 \mathrm{D}$ and $3 \mathrm{D}$ coordination networks have been demonstrated for a range of ligands (Carlucci et al., 1995) (Chen et al., 2006) (Roy et al., 2016).

Luminescent coordination polymers containing silver(I) were first documented in 1999 (Tong et al., 1999) with the report of a self-assembled 3D coordination polymer of silver(I) with bis-phenol Schiff base ligands that showed bright blue emission in both the solid state and in solution. Since then, many luminescent silver coordination polymers have been reported. Macrocycles and 2D structures made of $\mathrm{Ag}(\mathrm{I})$ and pyrimidine-based thioether ligands, reported by Han and coworkers (Han et al., 2005), also show bright luminescence. Liu and coworkers (Liu et al., 2005) reported 2D networks with metallocyclophane motifs with high electric conductivity due to columnar aromatic stacking motifs formed through both intra- and intermolecular $\pi-\pi$ interactions. They also report a 2D zigzag sheet structure, in which silver triflate forms tubelike double chains with $4,4^{\prime}$-bis $(2,5-$ dimethylstyryl) biphenyl molecules acting as links between the chains to form the sheets. Both of those compounds exhibit luminescence in the solid state and show excitation and emission maxima are shifted to longer wavelength as compared to those of the corresponding metal-free ligands. The same observation was made by Huo and coworkers (Huo et al., 2016) who reported a series of luminescent $\mathrm{Ag}(\mathrm{I})$ coordination polymers with different coordination modes tuned via different multidentate bis(1,2,3-triazole) ligands and anions. This anion-responsive behavior was also detected by Fan and coworkers (Fan et al., 2014) in 3D porous luminescent triazol-type Ag(I) framework with 
green luminescence emission. Co-crystallization of $\mathrm{Ag}(\mathrm{I})$ complexes with bipyridine and benzimidazole was reported by Cai and coworkers (Cai et al., 2011) to yield 1D infinite coordination polymer chains with both intraligand emission and ligand-to-metal charge transfer contribution. We reported the first example of a phosphorescent $\mathrm{Ag}(\mathrm{I})$ coordination polymer that incorporated iridium (III) metalloligands (Rota Martir et al., 2018).

The two benzene rings of the [2.2] paracyclophane (pCp) are disposed cofacially (Bahrin et al., 2017, 2), which give rise to through-space $(\pi-\pi$ trans-annular $)$ and through-bond $[\sigma$ (bridge $)-\pi$ (annular)] electronic interactions, affecting the chemical, optical, and electronic properties of the molecule (Kahnt et al., 2007; Elacqua and MacGillivray, 2010). It has been shown that positioning organic functional groups on the pCp core ring shifts its luminescence properties (Braun et al., 2017; Anhäuser et al., 2019; Rota Martir et al., 2019). Exploiting the electronic coupling present between the two benzene decks of the $\mathrm{pCP}$, the first examples of $\mathrm{pCp}$ compounds emitting via a thermally activated delayed fluorescence mechanism were recently reported by Spuling, Sharma and co-workers (Spuling et al., 2018). There are few examples of the pCp scaffold used in metallosupramolecular self-assembly (Meyer-Eppler et al., 2014; Gon et al., 2017; Anhäuser et al., 2019; Rota Martir et al., 2019). A $\left[\mathrm{Pd}_{3}(\mathrm{pCpd} 4 \mathrm{py})_{6}\right]\left(\mathrm{BF}_{4}\right)_{6}$ 3D-photoactive cage assembly incorporating a pyridyl-substituted $\mathrm{pCp}$ scaffold and $\mathrm{Pd}$ has been reported (Rota Martir et al., 2019), this macrocycle is emissive in both the solid state and solution, with emission red-shifted relative to the ligand.

Herein we report the synthesis and photoluminescence properties of a remarkable $1 \mathrm{D}$ coordination polymer containing silver(I) ions in two different coordination environments. The coordination polymer Ag-pCp was obtained from the reaction of $\mathrm{Ag}(\mathrm{I})$ ions and a [2.2] paracyclophane functionalized at the 7 and 15-positions with 4-pyridyl moieties (pCpd4py).

\section{METHODS}

\section{Synthesis}

The ligand pCpd4py was prepared according to a previously published method (Rota Martir et al., 2019). All other chemicals and solvents were obtained from commercial suppliers and used as received, solvents used for photophysical measurements were of spectroscopic grade.

Synthesis of $A g-p C p$ polymer. In a small vial, pCpd4py (4.0 mg, $0.01 \mathrm{mmol}, 1$ equiv.) was dissolved in $1 \mathrm{ml}$ of dichloromethane. To this vial a solution of $\mathrm{AgPF}_{6}(10 \mathrm{mg}$, $0.04 \mathrm{mmol}, 3.6$ equiv.) in $1 \mathrm{ml}$ of acetonitrile was carefully layered on top. The vial was sealed and left at room temperature, crystals began forming after 3 days and the reaction was complete after 10 days. The colorless crystals were collected to give the product $(5.0 \mathrm{mg}, 52 \%$ yield $)$. The product was identified by single crystal X-ray diffraction, with the formula determined to be $\left[\mathrm{Ag}_{3}(\mathrm{pCpd} 4 \mathrm{py})_{4}\left(\mathrm{NCCH}_{3}\right)_{6}\left(\mathrm{CH}_{2} \mathrm{Cl}_{2}\right)_{2}\right]$ (CCDC: 2089070).

\section{Photophysics}

Absorption spectra were recorded at room temperature on a Shimdazu-1800 spectrophotometer in $1 \mathrm{~cm}$ quartz cuvettes. For emission studies, degassed solutions were prepared via three freeze-pump-thaw cycles and spectra were taken using a home-made Schlenk quartz cuvette. Crystalline samples were finely ground and mounted in a quartz sample holder for solid-state measurements. Steady-state emission, excitation spectra and time-resolved emission spectra were recorded at $298 \mathrm{~K}$ using an Edinburgh Instruments F980 fluorimeter. Samples were excited at $378 \mathrm{~nm}$ using a pulsed diode laser for time-resolved measurements.

\section{RESULTS AND DISCUSSION}

Coordination of silver ions to the 4-pyridyl groups of pCpd4py proceeded readily, with the coordination polymer crystallizing from solution cleanly in moderate yield.

The X-ray crystal structure of the coordination polymer is shown in Figure 1. The asymmetric unit of the structure comprises one and a half silver(I) ions, two pCp4py ligands, one and a half $\mathrm{PF}_{6}{ }^{-}$anions, one molecule of dichloromethane solvent and three molecules of acetonitrile solvent. This remarkable structure consists of a 1D coordination polymer in which there are two different coordination environments for the silver(I) ions. One silver ion (Ag1) is in a linear, 2-coordinate environment, while the second silver ion (Ag2) is in a trigonal planar 3-coordinate environment.

The linear coordination environment around Ag1 has two strongly bound nitrogen atoms from the pCpd4py ligand. Ag1 is located at an inversion center, this results in a perfect $180^{\circ} \mathrm{N}-\mathrm{Ag} 1$ $\mathrm{N}$ bond angle and identical Ag1-N distances of 2.141(4) Å. A linear N-Ag-N bond has been observed in some Ag-coordination polymers, for example in rac-IrAg (Rota Martir et al., 2018); however, in many cases the linear coordination is distorted by the presence of anions and solvent molecules (Chen et al., 2006). In the extended coordination sphere, there are two weakly bound acetonitrile molecules, with longer Ag to $\mathrm{N}$ distances of 2.833(10) $\AA$. The weakly coordinated acetonitrile molecules are nearly perpendicular to the $A g-\mathrm{N}_{\mathrm{py}}$ bonds, with a $\mathrm{N}_{\mathrm{py}}-\mathrm{Ag} 1-\mathrm{N}_{\mathrm{MeCN}}$ angle of 87.6(3) The Agl-N distance in Ag-pCp is similar to the 2.141 and $2.156 \AA$ observed in rac-IrAg (Rota Martir et al., 2018) and also similar to the Ag-N distances of between 2.123 and $2.133 \AA$ seen in a series of linear two-coordinate $\left.[\mathrm{Ag} \text { (pyridine) })_{2}\right]^{+}$ structures. (Chen et al., 2007).

The coordination environment around Ag2 is a nearly perfect trigonal planar environment, with Ag2-N bond lengths of 2.224(4), 2.246(5) and 2.268(4) $\AA$ and $\mathrm{N}-\mathrm{Ag} 2-\mathrm{N}$ angles between 112.85(16) and 124.31(17), with the $\operatorname{Ag}(\mathrm{I})$ ion displaced $0.099 \AA$ out of the mean-plane of the nitrogen atoms. This is similar to the coordination environment seen in a pair of $\left[\mathrm{Ag}(\text { isonicotinamide })_{3}\right]^{+}$crystals where $\mathrm{Ag}-\mathrm{N}$ bond lengths ranged between 2.213 and $2.321 \AA, \mathrm{N}-\mathrm{Ag}-\mathrm{N}$ bond angles were $c a .105,120$ and $135^{\circ}$, and the $\operatorname{Ag}(\mathrm{I})$ ion was displaced 0.007 or $0.017 \AA$ out of the mean-plane of the nitrogen atoms (B. Aakeröy et al., 1998). A three-coordinate 


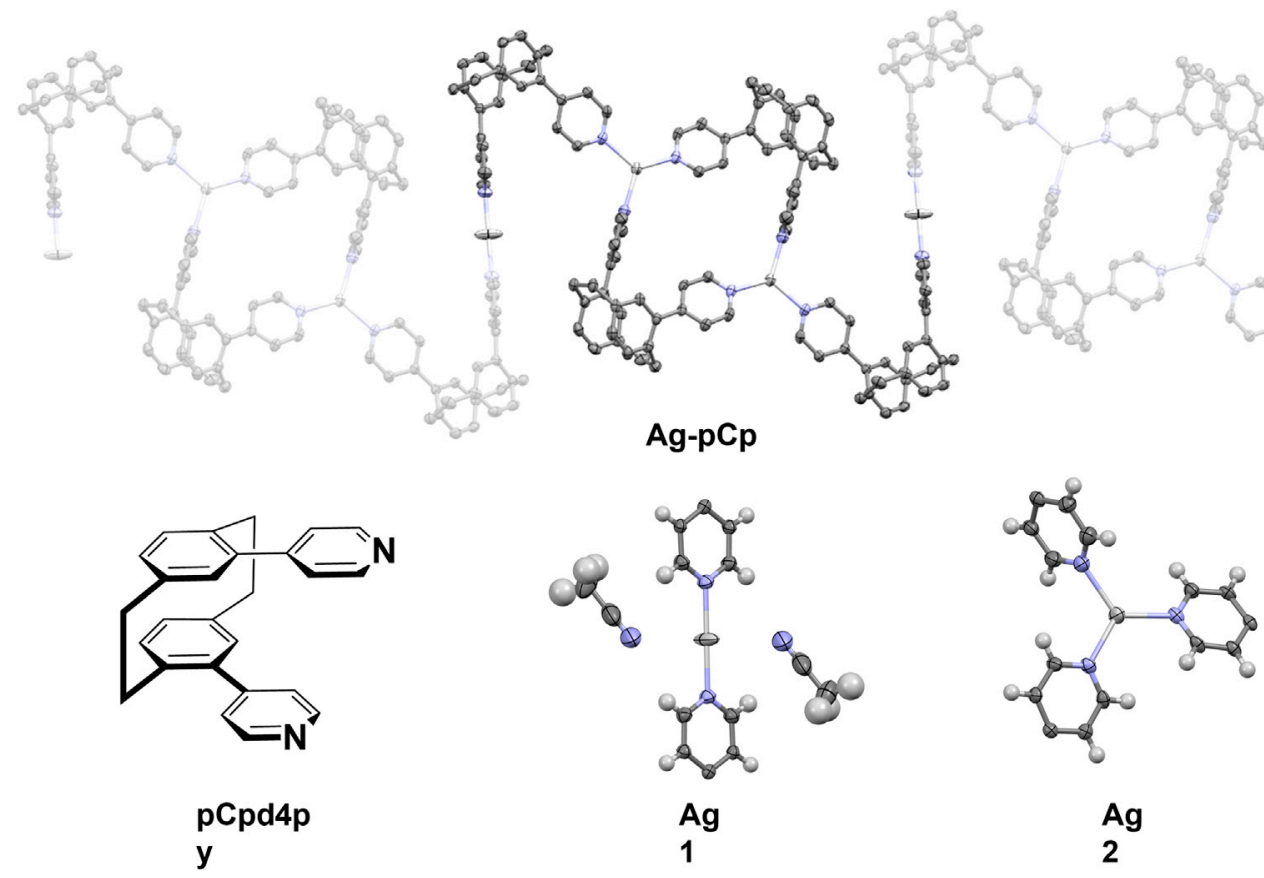

FIGURE 1 | (Top) Representation of the X-ray crystal structure of Ag-pCp, hydrogen atoms, solvent molecules and anions are omitted for clarity. (Bottom, L-R) Diagram of ligand pCpd4py, two different coordination environments for the silver atoms (thermal ellipsoid plot, $50 \%$ probability ellipsoids).

geometry for silver is the third most common seen, behind fourand two-coordinate (Young and Hanton, 2008), in many of these examples the coordination environment is distorted from the ideal trigonal planar, trigonal pyramidal or T-shaped geometries by the use of bidentate ligands or the presence of weak interactions with solvent molecules or anions (Durini et al., 2017). The angled orientation of the coordinating pyridyl groups makes each Ag2 center a chiral molecular propeller, although from the symmetry of the space group, centers of both handedness are present and individual polymer chains are achiral.

The phenyl rings of the pCp4py ligands show a distortion from planarity similar to that seen in related pCp compounds (angle across the para-substitution axis 15.6-16.2 ${ }^{\circ}$ ). (Rota Martir et al., 2019). Despite this, the dihedral angle between mean planes of the phenyl groups still shows them to be parallel $\left(1.8^{\circ}\right.$ for both ligands). Each ligand shows two rather different dihedral angles between the phenyl and its pyridyl substituent, although these match reasonably well between the two ligands (31.6 and $49.2^{\circ}$ for one, 36.9 and $51.7^{\circ}$ for the other). In turn, this leads to similar angles between pyridyl rings for each of the two ligands (75.4 and $83.2^{\circ}$ ). This matches the conformation seen in other pyridyl-cyclophanes. (Rota Martir et al., 2019).

Each polymer chain is built from alternating $\left[\mathrm{Ag}_{2}(\mathrm{pCpd} 4 \mathrm{py})_{2}\right]$ 32-membered metallamacrocycles (which include just the trigonal planar Ag2) and Z-shaped linkers, comprising two ligands bound linearly to Ag1. This results in a flat, tape-like coordination polymer (Figure 1), with chains propagating along the $\left[\begin{array}{lll}0 & 1 & -1\end{array}\right]$ diagonal axis, and a polymer repeat-distance of $21.42 \AA$. Adjacent polymer chains are offset such that the edge of one tape overlies that of the next, with the pCp of the Z-shape above the trigonal planar Ag2 center. Despite this positioning, no $\pi \cdots \pi$ interactions are found in the structure, the shortest distance between centroids being $4.33 \AA$. However, this positioning of adjacent chains does allow for the formation of $\mathrm{CH} \cdots \pi$ interactions chains, involving a methylene hydrogen of one pCpd4py and the $\pi$-system of a pyridine ring of the other, at a C-H...centroid distance of $2.70 \AA$ [C...centroid separation of $3.528(6) \AA]$. These interactions occur in pairs across an inversion center, and link adjacent chains into two-dimensional sheets in the ( $\left.\begin{array}{lll}1 & 1 & 1\end{array}\right)$ plane (Figure 2). No direct intermolecular interactions occur between adjacent sheets. There are no close contacts between the silver(I) atoms, in contrast to many lowcoordinate silver complexes. (Chen et al., 2007).

Both anions and solvent molecules form weak C-H...A interactions, although only the $\mathrm{PF}_{6}^{-}$anions and one of the molecules of acetonitrile interact directly with the polymer chains, other interactions being between anion and solvent or solvent and solvent. None of the anions or solvent molecules bridge directly between chains. The $\mathrm{C}-\mathrm{H} \cdots \mathrm{N}$ distances occur at 2.47 and $2.61 \AA[\mathrm{C} \cdots \mathrm{N}$ separations of $3.371(15)$ and $3.24(2) \AA]$ and the $\mathrm{C}-\mathrm{H}$... F distances range from 2.40 to $2.45 \AA$ [corresponding $\mathrm{C} \cdots \mathrm{F}$ separations of 3.150(9) to $3.400(14) \AA$ ] . One $\mathrm{PF}_{6}{ }^{-}$interacts solely with one polymer chain, while the other interacts with a chain and multiple solvent molecules. These weak interactions give rise to discrete T-shaped motifs comprising one dichloromethane, one $\mathrm{PF}_{6}{ }^{-}$and three acetonitriles (Figure 2). Two polymer chains in the same sheet are weakly linked via the $\mathrm{PF}_{6}{ }^{-}$and one acetonitrile, while this sheet can in turn be linked to a chain in another sheet via the weak Ag...NCMe interactions. 

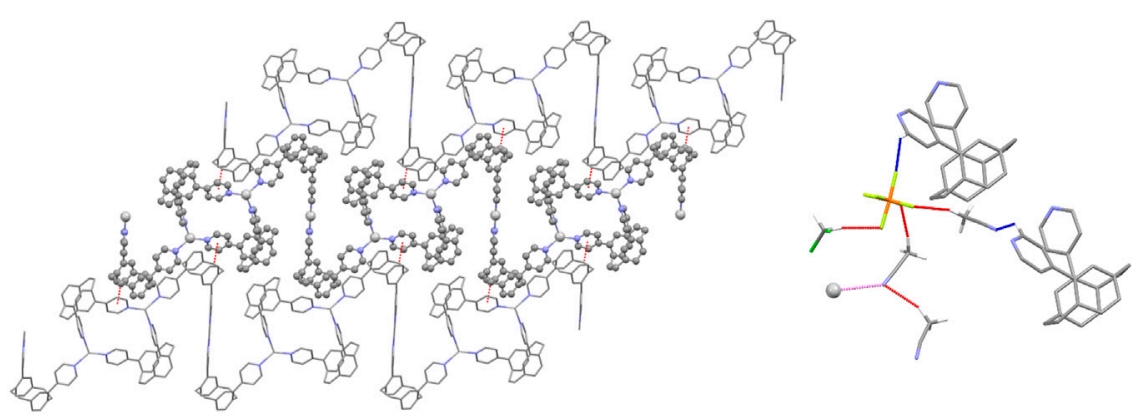

FIGURE 2 | Representations of the intermolecular interactions in the solid-state structure of Ag-pCp. (Left) View of a two-dimensional sheet in the (1 1 1) plane formed by $\mathrm{C}-\mathrm{H} \cdot \pi$ interactions (shown red) linking adjacent chains. The central chain is highlighted for clarity. (Right) View of the weakly interacting T-shaped motif (interactions shown red) formed by solvent and anion. Links from the motif to polymer chains are shown for both $\mathrm{C}-\mathrm{H} \ldots \mathrm{A}$ (shown blue) and Ag... N (shown pink) interactions
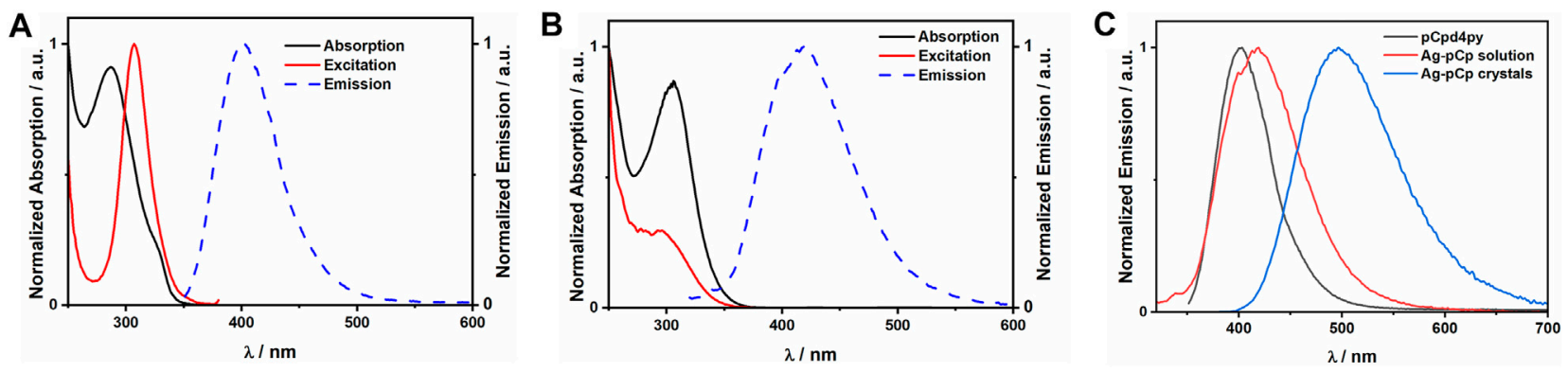

FIGURE 3|Plots of the normalized absorption, excitation and emission spectra for (A) pCpd4py in acetonitrile, (B) Ag-pCp in dichloromethane. (C) Comparison of the emission spectra for the ligand and $\mathrm{Ag}-\mathrm{pCp}$ in solution and crystalline states.

Ag-pCp is luminescent, exhibiting sky-blue emission $\left(\lambda_{\mathrm{PL}}=497 \mathrm{~nm}\right)$ in the crystalline solid state and deep-blue emission in DCM solution $\left(\lambda_{\mathrm{PL}}=419 \mathrm{~nm}\right)$, Figure 3 . Coordination polymers are liable to exist as shorter oligomers in solution, although in a non-coordinating solvent like DCM, $\mathrm{Ag}-\mathrm{pCp}$ is expected to retain its structural motifs in the oligomeric fragments. The solution-state emission is only slightly red-shifted from the emission of the ligand $\left(\lambda_{\mathrm{PL}}=\right.$ $403 \mathrm{~nm}$ ), suggesting that the emission is fluorescence from a ligand centered $\left({ }^{1} \mathrm{LC}\right)$ singlet excited state, with the red-shift due to the stabilization of the LUMO and the singlet excited state upon coordination of the Lewis acidic silver(I) ions to the pyridyl group of the ligand. This same red-shift in emission upon coordination of a metal ion to $\mathrm{pCp}-\mathrm{d} 4 \mathrm{py}$ was seen for $\left[\mathrm{Pd}_{3}(\mathrm{pCpd} 4 \mathrm{py})_{6}\right]\left(\mathrm{BF}_{4}\right)_{6}$. (Rota Martir et al., 2019). There is a similar red-shift observed in the absorption spectrum of a DCM solution of $\mathrm{Ag}$-pCp $\left(\lambda_{\mathrm{bss}}=306 \mathrm{~nm}\right)$ in comparison to the free ligand $\left(\lambda_{\mathrm{abs}}=287 \mathrm{~nm}\right)$. The emission lifetime of pCpd4py and the DCM solution of Ag-pCp are very similar (pCpd4py: $\tau_{\mathrm{PL}}=$ 5.42 ns, Ag-pCp: $\tau \mathrm{P}_{\mathrm{L}}=5.27 \mathrm{~ns}$ ), while crystalline Ag-pCp has a longer emission lifetime of $19 \mathrm{~ns}$.

The electronic properties of both the ligand and subunits of the polymer were investigated by DFT and TD-DFT calculations to provide further insight into the nature of the emission properties and the corresponding energy levels of the excited states of the compounds. Details of the calculations performed are provided in the Supplementary Material, while plots of the Kohn-Sham orbitals are shown in Figure 4. The optimized structure of the ligand showed a large gap of $4.50 \mathrm{eV}$ between the HOMO and LUMO, which is also reflected in the high excited state energy of $3.77 \mathrm{eV}$ for $\mathrm{S}_{1}$. There is no spatial separation between the HOMO and LUMO, leading to a large singlet-triplet energy gap, $\Delta \mathrm{E}_{\mathrm{ST}}$, of $0.89 \mathrm{eV}$.

We next investigated Ag-pCp. The polymer was broken down into two different chromophoric units that were studied separately. The first unit contained a single two-coordinate silver center with the two coordinating pCpd4py ligands (matching the environment of Ag1). The second unit contained two three-coordinate silver centers, with two pCpd4py ligands bridging the Ag ions and forming a metallamacrocycle, and two additional ligands completing the coordination sphere of each Ag center (matching Ag2). The optimized geometry for each silver environment is similar to the crystallographically determined structures. The optimized Ag1 structure has a linear, two-coordinate environment around the silver ion, with the two pyridine groups being co-planar. The Ag2 structure retains the trigonal planar coordination environment observed experimentally, although the environment is slightly distorted, with py-Ag-py bond angles ranging from 94 to $140^{\circ}$ and the $A g-\mathrm{N}_{\text {py }}$ distances ranging from 2.238 to $2.395 \AA$ with the silver ions displaced up to $0.229 \AA$ from the mean-plane of the nitrogen atoms. 


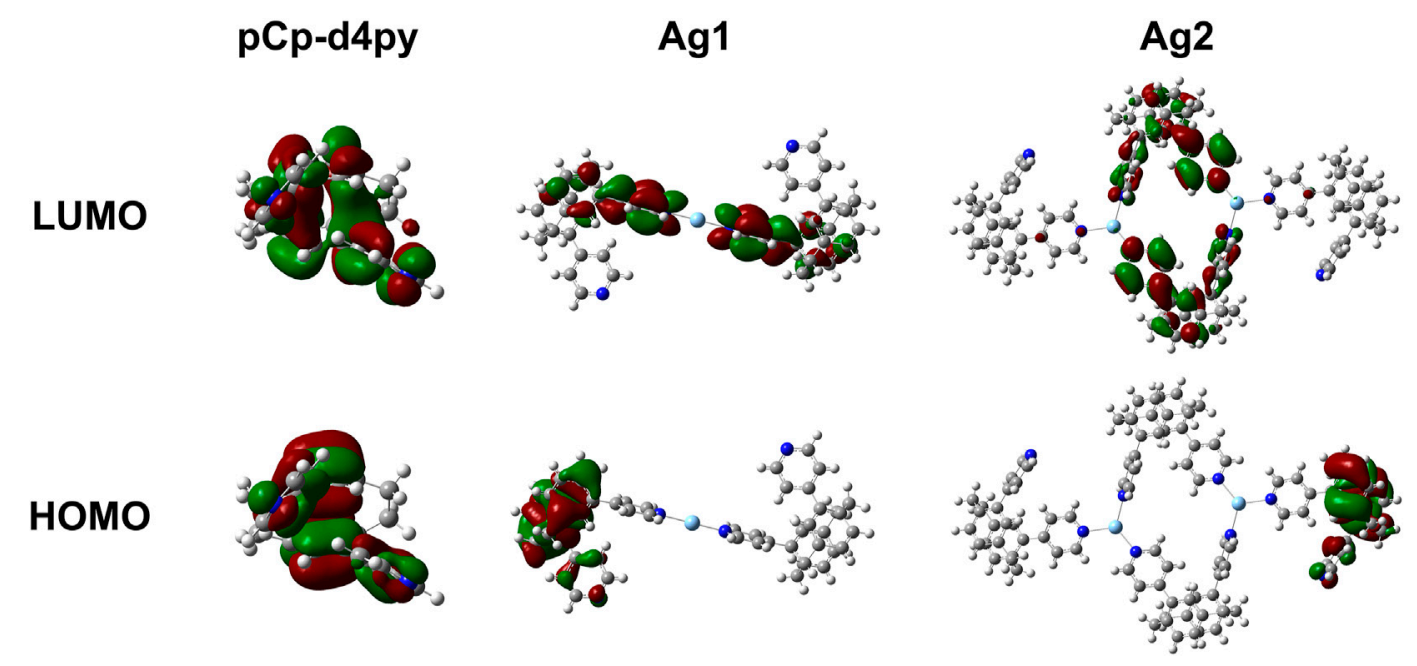

FIGURE 4 | Plots of the molecular orbitals (top-LUMO, bottom-HOMO) for pCpd4py and the two different Ag-containing model units from Ag-pCp.

The coordination of the silver ions to the ligand in each of these units resulted in a strong stabilization of more than $50 \mathrm{meV}$ on the LUMO of the compounds, with little change observed in the HOMO energy. The HOMO remaining localized on a single ligand, while the LUMO is distributed across multiple ligands. In all cases there is no metal contribution to the orbitals (Figure 3).

The smaller Ag1-type unit shows a significant (50 meV) red-shift in the excited state energy of $S_{1}$, while the larger Ag2type unit shows a more moderate $20 \mathrm{meV}$ red-shift of the emissive excited state. The calculated red-shift for the Ag2containing fragment is close to the $11 \mathrm{meV}$ red-shift observed between the solution state emission of the ligand in $\mathrm{MeCN}$ and that of the polymer in DCM. This suggests that the Ag2-type group is a good computational model for the whole polymer in solution.

\section{CONCLUSION}

We have prepared a new luminescent $1 \mathrm{D}$ silver(I) coordination polymer Ag-pCp. The polymer has a very interesting structure, containing both linear 2-coordinate and trigonal planar 3-coordinate silver atoms in the polymer chain. Ag-pCp is blue fluorescent in both the crystalline state and in DCM solution.

\section{REFERENCES}

Aakeröy, C. B., Beatty, A. M., and Helfrich, B. A. (1998). Two-fold Interpenetration of 3-D Nets Assembled via Three-Co-Ordinate Silver(I) Ions and AmideAmide Hydrogen Bonds. J. Chem. Soc. Dalton Trans., 1943-1946. doi:10.1039/ A802520G

Anhäuser, J., Puttreddy, R., Lorenz, Y., Schneider, A., Engeser, M., Rissanen, K., et al. (2019). Chiral Self-Sorting Behaviour of [2.2]paracyclophane-Based

\section{DATA AVAILABILITY STATEMENT}

The datasets presented in this study can be found in online repositories. The names of the repository/repositories and accession number(s) can be found below: https://doi.org/10.17630/ 9e0d4b83-171a-4805-aef8-a9fbac035clf.

\section{AUTHOR CONTRIBUTIONS}

CM, LD, RMD, DC, AS, and EZ-C managed the project and coauthored the manuscript.

\section{FUNDING}

$\mathrm{CM}$ and EZ-C wish to thank the Engineering and Physical Sciences Research Council (EP/P014082/1, EP/M02105X/1 and EP/ R035164/1).

\section{SUPPLEMENTARY MATERIAL}

The Supplementary Material for this article can be found online at: https:/www.frontiersin.org/articles/10.3389/fchem.2021.728845/ full\#supplementary-material

Bis(pyridine) Ligands. Org. Chem. Front. 6, 1226-1235. doi:10.1039/ C9QO00155G

Bahrin, L. G., Sarbu, L. G., Jones, P. G., Birsa, L. M., and Hopf, H. (2017). [2.2] Paracyclophane-bis(triazole) Systems: Synthesis and Photochemical Behavior. Chem. Eur. J. Eur. J. 23, 12338-12345. doi:10.1002/chem.201701593

Braun, C., Nieger, M., and Bräse, S. (2017). Unprecedented One-Pot Reaction towards Chiral, Non-racemic Copper(I) Complexes of [2.2]ParacyclophaneBased P,N -Ligands. Chem. Eur. J. 23, 16452-16455. doi:10.1002/ chem.201704115 
Cai, S.-L., Zheng, S.-R., Tan, J.-B., Pan, M., Fan, J., and Zhang, W.-G. (2011). An Unprecedented Supramolecular Network with Channels Filled by 1D Coordination Polymer Chains: Cocrystallization of Ag(i)-4,4'-Bipyridine and Ag(i)-Benzimidazole Complexes. CrystEngComm 13, 6345-6348. doi:10.1039/C1CE05535F

Carlucci, L., Ciani, G., Proserpio, D. M., and Sironi, A. (1995). 1-, 2-, and 3Dimensional Polymeric Frames in the Coordination Chemistry of AgBF4 with Pyrazine. The First Example of Three Interpenetrating 3-Dimensional Triconnected Nets. J. Am. Chem. Soc. 117, 4562-4569. doi:10.1021/ja00121a014

Chen, C.-L., Kang, B.-S., and Su, C.-Y. (2006). Recent Advances in Supramolecular Design and Assembly of Silver(i) Coordination Polymers. Aust. J. Chem. 59, 3-18. doi:10.1071/ch05225

Chen, C. Y., Zeng, J. Y., and Lee, H. M. (2007). Argentophilic Interaction and Anionic Control of Supramolecular Structures in Simple Silver Pyridine Complexes. Inorg. Chim. Acta 360, 21-30. doi:10.1016/j.ica.2006.06.013

Durini, S., Ardizzoia, G. A., Therrien, B., and Brenna, S. (2017). Tuning the Fluorescence Emission in Mononuclear Heteroleptic Trigonal Silver(i) Complexes. New J. Chem. 41, 3006-3014. doi:10.1039/C6NJ04058F

Elacqua, E., and MacGillivray, L. R. (2010). From the Decks to the Bridges: Optoelectronics in [2.2] paracyclophane Chemistry. Eur. J. Org. Chem. 2010, 6883-6894. doi:10.1002/ejoc.201000930

Fan, Z., Wang, Y. Y., Xu, Y. Y., Su, X. M., Wu, X. X., Huo, J. Z., et al. (2014). Synthesis and Characterization of a Novel 3D Porous Luminescent $\mathrm{Ag}(\mathrm{I})$ Framework with a Multidentate Triazole Ligand. Inorg. Chim. Acta 410, 178-182. doi:10.1016/j.ica.2013.11.002

Gon, M., Morisaki, Y., and Chujo, Y. (2017). A Silver(I)-induced Higher-Ordered Structure Based on Planar Chiral Tetrasubstituted [2.2]paracyclophane. Chem. Commun. 53, 8304-8307. doi:10.1039/C7CC03615A

Han, L., Wu, B., Xu, Y., Wu, M., Gong, Y., Lou, B., et al. (2005). Assembly of Luminescent $\operatorname{Ag}(\mathrm{I})$ Coordination Architectures Adjusted by Modification of Pyrimidine-Based Thioether Ligands. Inorg. Chim. Acta 358, 2005-2013. doi:10.1016/j.ica.2004.12.023

Huo, J. Z., Su, X. M., Wu, X. X., Liu, Y. Y., and Ding, B. (2016). Hydrothermal Synthesis and Characterization of a Series of Luminescent $\mathrm{Ag}(\mathrm{I})$ Coordination Polymers with Two New Multidentate Bis-(1,2,3-Triazole) Ligands: Structural Diversity, Polymorphism and Photoluminescent Sensing. Cryst. Eng. Comm. 18, 6640-6652. doi:10.1039/C6CE01275B

Kahnt, A., Guldi, D. M., de la Escosura, A., Martínez-Díaz, M. V., and Torres, T. (2007). [2.2]Paracyclophane: a Pseudoconjugated Spacer for Long-Lived Electron Transfer in Phthalocyanine-C60dyads. J. Mater. Chem. 18, 77-82. doi:10.1039/B712751K

Liu, S. Q., Kuroda-Sowa, T., Konaka, H., Suenaga, Y., Maekawa, M., Mizutani, T., et al. (2005). Silver(I) Coordination Polymers of Fluorescent Oligo(phenylenevinylene) with $\pi-\pi$ Stackings: Luminescence and Conductivity. Inorg. Chem. 44, 1031-1036. doi:10.1021/ic0400585

Medici, S., Peana, M., Crisponi, G., Nurchi, V. M., Lachowicz, J. I., Remelli, M., et al. (2016). Silver Coordination Compounds: A New Horizon in Medicine. Coord. Chem. Rev. 327-328, 349-359. doi:10.1016/j.ccr.2016.05.015

Meyer-Eppler, G., Topić, F., Schnakenburg, G., Rissanen, K., and Lützen, A. (2014). Chiral Self-Sorting Oftrans-Chelating Chiral Ligands upon Formation of PdIIComplexes. Eur. J. Inorg. Chem. 2014, 2495-2501. doi:10.1002/ejic.201402057

Rota Martir, D., Delforce, L., Cordes, D. B., Slawin, A. M. Z., Warriner, S. L., Jacquemin, D., et al. (2019). A Pd3L6 Supramolecular Cage Incorporating Photoactive [2.2]paracyclophane Units. Inorg. Chem. Front. 7, 232-238. doi:10.1039/C9QI01147A
Rota Martir, D., Rajamalli, P., Cordes, D. B., Slawin, A. M. Z., and Zysman-Colman, E. (2018). Marigold Flower-like Assemblies of Phosphorescent Iridium-Silver Coordination Polymers. Macromol. Rapid Commun. 39, 1800501. doi:10.1002/ marc.201800501

Roy, S., Titi, H. M., Tripuramallu, B. K., Bhunia, N., Verma, R., and Goldberg, I. (2016). Silver Coordination Polymers Based on Newly Designed Bis(cyanobenzyl)bipiperidine Ligand: Synthesis, Anion Exchange, Guest Inclusion, Electrochemical, and Photoluminescence Properties. Cryst. Growth Des. 16, 2814-2825. doi:10.1021/acs.cgd.6b00151

Spuling, E., Sharma, N., Samuel, I. D. W., Zysman-Colman, E., and Bräse, S. (2018). (Deep) Blue Through-Space Conjugated TADF Emitters Based on [2.2] paracyclophanes. Chem. Commun. 54, 9278-9281. doi:10.1039/C8CC04594A

To, W.-P., Cheng, G., Tong, G. S. M., Zhou, D., and Che, C.-M. (2020). Recent Advances in Metal-TADF Emitters and Their Application in Organic LightEmitting Diodes. Front. Chem. 8, 653. doi:10.3389/fchem.2020.00653

Tong, M.-L., Chen, X.-M., Ye, B.-H., and Ji, L.-N. (1999).Self-Assembled ThreeDimensional Coordination Polymers with Unusual Ligand-Unsupported Ag-Ag Bonds: Syntheses, Structures, and Luminescent Properties. Angew. Chem. Int. Ed. 38, 2237-2240. doi:10.1002/(SICI)1521-3773(19990802)38: $15<2237::$ AID-ANIE2237>3.010.1002/(sici)1521-3773(19990802)38:15<2237:: aid-anie2237>3.0.co;2-9

Yang, L., Tan, X., Wang, Z., and Zhang, X. (2015). Supramolecular Polymers: Historical Development, Preparation, Characterization, and Functions. Chem. Rev. 115, 7196-7239. doi:10.1021/cr500633b

Yersin, H., Czerwieniec, R., Shafikov, M. Z., and Suleymanova, A. F. (2017). TADF Material Design: Photophysical Background and Case Studies Focusing on CuI and AgI Complexes. ChemPhysChem 18, 3508-3535. doi:10.1002/cphc.201700872

Yi, F.-Y., Chen, D., Wu, M.-K., Han, L., and Jiang, H.-L. (2016). Chemical Sensors Based on Metal-Organic Frameworks. ChemPlusChem 81, 675-690. doi:10.1002/cplu.201600137

Young, A. G., and Hanton, L. R. (2008). Square Planar Silver(I) Complexes: A Rare but Increasingly Observed Stereochemistry for Silver(I). Coord. Chem. Rev. 252, 1346-1386. doi:10.1016/j.ccr.2007.07.017

Zeng, L., Guo, X., He, C., and Duan, C. (2016). Metal-Organic Frameworks: Versatile Materials for Heterogeneous Photocatalysis. ACS Catal. 6, 7935-7947. doi:10.1021/acscatal.6b02228

Conflict of Interest: The authors declare that the research was conducted in the absence of any commercial or financial relationships that could be construed as a potential conflict of interest.

Publisher's Note: All claims expressed in this article are solely those of the authors and do not necessarily represent those of their affiliated organizations, or those of the publisher, the editors and the reviewers. Any product that may be evaluated in this article, or claim that may be made by its manufacturer, is not guaranteed or endorsed by the publisher.

Copyright (C) 2021 Mackenzie, Delforce, Rota Martir, Cordes, Slawin and ZysmanColman. This is an open-access article distributed under the terms of the Creative Commons Attribution License (CC BY). The use, distribution or reproduction in other forums is permitted, provided the original author(s) and the copyright owner(s) are credited and that the original publication in this journal is cited, in accordance with accepted academic practice. No use, distribution or reproduction is permitted which does not comply with these terms. 